\title{
Hospital stay of inpatients HIV/AIDS with mental and behavioral disorders: 2007 vs 2010 discharges in the United States
}

\author{
Priscilla O. Okunji ${ }^{* 1}$, Johnnie Daniel ${ }^{2}$ \\ ${ }^{1}$ Division of Nursing, College of Nursing and Allied Health Sciences, Howard University, Washington, DC, United States \\ ${ }^{2}$ Department of Sociology and Anthropology, Howard University, Washington, DC, United States
}

Received: September 25, 2014

Accepted: December 9, 2014 Online Published: January 11, 2015

DOI: $10.5430 /$ jnep.v5n3p110

URL: http://dx.doi.org/10.5430/jnep.v5n3p110

\begin{abstract}
About 1.1 million Americans are living with HIV, and 21\% of these persons do not know they are infected. African Americans are 8.6 times more likely to be diagnosed with HIV infection, as compared to the White population in 2008. Women account for a $25 \%$ of all new HIV/AIDS cases, with approximately $65 \%$ having contracted the infection via heterosexual contact. People with mental and behavioral disorders are at increased risk for HIV/AIDS. Tendencies for committing risky behavior that leads to HIV infection is around 50\% among people who are mentally ill. Current nation-based statistics of factors that affect the outcomes of inpatients HIV/AIDS admitted to non-federal hospitals is lacking. A retrospective data analysis of discharges was conducted from the 2007 and 2010 Healthcare Cost and Utilization Project (HCUP) Nationwide Inpatient Sample (NIS). Sample selection criteria was done using the International Classification of Diseases, Ninth Revision (ICD-9) codes (HIV/AIDS $=042$, Schizophrenia $=295$, Depression $=296$, Bipolar $=657$ ). Statistical Analysis involved the use of crosstab, and multiple regression for patient measures (age, gender, ethnicity, income, insurance, patients' co-morbidities, admission types, treatment procedures); hospital measures (teaching status, ownership, location, region, size) and Length Of Stay (LOS). The result of this study has shown that the patient demographics age, gender, ethnicity, insurance, location and size or region of the hospitals were not the most critical variables that made the patients stay longer on admission to non-federal hospitals. These findings highlight the conversation that sicker patients with more comorbidities are usually admitted to teaching hospitals where more Registered Nurses (RNs) are employed full time for a more positive and satisfactory patient outcomes. Finally, the result confirmed the relationship between the ratio of RNs FTEs and patient LOS. This has clinical implications in hiring, retention and expectations as it regards to inpatients HIV/AIDS outcomes.
\end{abstract}

Key Words: Inpatients, HIV/AIDS, Schizophrenia, Bipolar, Depression, Discharges, Length of stay

\section{Introduction}

A continued increase in HIV/AIDS infections among minorities and the underserved is one of the greatest concerns to HIV/AIDS researchers across the country. The Center for Disease Control and Prevention (CDC) estimates in 2014 that about 1.1 million Americans are living with HIV, and that $21 \%$ of these persons do not know they are infected. ${ }^{[1]}$ African Americans are 8.6 times more likely to be diagnosed with HIV infection, as compared to the White population in 2011. ${ }^{[2]}$ In the general population, women account for a $25 \%$ of all new HIV/AIDS cases, with approxi-

\footnotetext{
${ }^{*}$ Correspondence: Priscilla O. Okunji; Email: Priscilla.okunji@ howard.edu; Address: Division of Nursing, College of Nursing and Allied Health Sciences, Howard University, Washington, DC, United States.
} 
mately $65 \%$ having contracted the infection via heterosexual contact. The synergy between substance and alcohol abuse and HIV transmission is extant in the literature; and compounded with mental illnesses like depression, bipolar disease and schizophrenia is an enormous challenge for HIV prevention research. People with mental and behavioral disorders are at increased risk for HIV/AIDS. ${ }^{[2]}$ Tendencies for committing risky behavior that leads to HIV infection is around $50 \%$ among people who are mentally ill. ${ }^{[2]}$ Individuals with high levels of HIV/AIDS specific symptoms do also report significantly higher substance-use behaviors, including injection drug use and heavy alcohol use. ${ }^{[3,4]}$ Study of this disease burden has been carried out primarily at the individual level due to the lack of data sources linking individual information to medical and mental health characteristics with inpatient data. ${ }^{[5]}$ Current pattern of the association between mental illness and behavioral factors among HIV/AIDS hospitalized patient with their health outcomes in non-federal hospitals is lacking. Reliable estimates of the variables are needed to determine the health outcome predictors and accurate analysis of costs to efficiently target limited resources for the greatest public health benefit of inpatients HIV/AIDS with mental illness and behavioral disorders. Our goal is to conduct a national estimate of the prevalence and factors affecting Length Of Stay (LOS) of inpatient HIV/AIDS adults with mental illness (schizophrenia, bipolar and depression) and behavioral disorders (alcohol and drug abuse) by determining the variables that are more predictive of the outcomes than the traditional HIV prognostic variables.

\section{Background}

While the US population is reported to exhibit increased HIV/AIDS incidence rates and mental disorders, limited information was found on the association between mental health and behavioral disorders on patient outcomes among hospitalized HIV/AIDS adults in non- federal hospitals. Research studies of this disease burden has been carried out primarily at the individual level due to the lack of national data sources linking individual information to medical and mental health characteristics with hospitals data. ${ }^{[5]}$ Additionally, the disproportionate burden of HIV/AIDS among minority population groups is well-known and documented. Further, adults who have mental illnesses and behavioral disorders, such as substance and alcohol use, make them vulnerable and places them at increased risk for HIV infection as well as delay to access HIV/AIDS care services. Optimal mental health is a critical component of HIV care maintenance. HIV/AIDS patients who have mental illnesses or behavioral disorders fail to comply with HIV treatment interventions resulting in dire public health consequences such as non-adherence to antiretroviral (ARV) medications leading to ARV drug resistance and further transmission of the HIV. The intersection of HIV/AIDS, mental illness,

Published by Sciedu Press and behavioral disorders also cause low productivity, which would have serious economic implications at the state and national levels if not addressed. Therefore, it is critical that this study is conducted to determine target variables that would help inform sustainable HIV prevention interventions that could decrease HIV transmission rates and infection. Our study is significant in important ways. It will help: (1) determine important mental and behavioral variables using population-based sample that contribute to inpatients HIV/AIDS LOS, to inform sustainable prevention efforts; (2) identify patient and hospital characteristics that substantially impact health outcomes in HIV/AIDS patients, so that appropriate health care services can be mobilized in areas that HIV/AIDS patients are most vulnerable and would yield stronger effect for positive patient care outcomes; and (3) determine the outcome predictors for the greatest public health benefit of inpatients HIV/AIDS with mental and behavioral diseases.

Mental disorders, such as bipolar disorder, schizophrenia, and depression, have been associated with both HIVassociated risk behaviors and HIV infection. ${ }^{[5]}$ Studies have shown that HIV may be increased among those with severe mental disorders, but this has been studied in only a limited number of sites. It has been reported that across 102 metropolitan statistical areas (MSAs), $1.81 \%$ of patients with schizophrenia had received diagnoses of HIV/AIDS. ${ }^{[5]}$ MSA rates ranged widely, from $5.2 \%$ in Newark, New Jersey, to no cases in 16 of the MSAs. Severe mental illness is associated with an increase in HIV risk. ${ }^{[4]}$ Pharmacologic treatments have been used for the management of HIV/AIDS and severe mental illness over the years have changed the prognosis and quality of life for millions of patients throughout the world. ${ }^{[6]}$ Highly active antiretroviral therapy (HAART) has altered AIDS epidemic in many countries and better-tolerated treatment regimens have resulted in many patients with HIV living longer and with an improved quality of life. In patients with severe mental illness, second generation antipsychotics have provided hope for many patients and families struggling with schizophrenia and bipolar disorder. However, despite these advances in treatment, metabolic abnormalities, metabolic syndrome kept occurring at a greater incidence in persons with both HIV and severe mental illness.

Patients with severe mental disorders are increasingly being diagnosed with HIV/AIDS, and higher prevalence rates of severe mental illness are been observed in HIV patients than the general population. Tendencies for indulging in risky behavior that leads to HIV contraction is around $50 \%$ in those people who are mentally ill. ${ }^{[2]}$ HIV prevalence in patients with mental disorders and out patients is $23 \%$ compared to just $0.4 \%$ for the rest of the population. ${ }^{[2]} \mathrm{Un}$ healthy substance-use behaviors that HIV-positive individuals engaged in may include heavy alcohol intake, illicit drug use, and cigarette smoking. Some of these behaviors are by 
many as a way to manage the disease-related symptoms. ${ }^{[7]}$ Relatively little research has focused on the role of serious mental illness (SMI) among injection drug users (IDUs) and non-IDUs in susceptibility to and transmission of HIV and there are few published reports on HIV risk among Latino with SMI ${ }^{[8]}$ Many studies have ascertained that poor mental health may be attributed to increased HIV risks. Conversely, mental and behavioral disorders may also have direct effect on the patient's mental health. ${ }^{[2]}$ Many HIV patients, especially those with childhood sexual abuse histories may continue to abuse drugs. ${ }^{[9]}$

Behaviors such as drug abuse are believed to relieve individuals from momentarily pain. Hence addict person may contract HIV through IV drug use via unclean syringes that may leave the risk of HIV when contaminated by infected blood. In a prospective cohort study factors associated with receipt of substance abuse treatment (SAT) among adults with alcohol problems and HIV/AIDS indicated need for SAT among people with HIV and alcohol problems (e.g., drug dependence) are associated with receipt of treatment. However, non-need factors (e.g., sexual orientation, age) that should not decrease the probability of receipt of treatment were identified as well in the study. ${ }^{[10]}$

Inconsistent condom use for vaginal sex with casual partners was reported among substance users $(43 \%)$ and incarcerated participants $(60 \%)$, whereas $26 \%$ of men who have sex with men reported inconsistent condom use for anal sex with casual partners. ${ }^{[4]}$ The study reported that overall, $56 \%$ and $29 \%$ sex with casual partners under the influence of alcohol and drugs in the past 6 months, respectively. The authors recommend that substance use issues in relation to sexual risk behaviors be addressed in future research. Measurement of risk behaviors has been used to guide tailored risk reduction strategies as HIV infection patterns shift toward rural minorities, particularly in regions where HIV disease remains highly stigmatized. ${ }^{[11]}$ Interactive Voice Response (IVR) systems has been used to enhance reports of sensitive behaviors in such areas and telehealth applications was used to extend the reach of care in rural, underserved areas. ${ }^{[11]}$

Sexual contact with multiple partners either male or female is a risky behavior for contracting HIV/AIDS. Steady single partner, irrespective of his sexual orientation is mostly harmless with more mental stability. Conversely having multiple partners may result into frustration, perversion etc, that in many cases may be counted as mental disorders such as depression. These acts increase the risk of HIV. Sexual abuse among men mostly, who indulge in sexual abuses and rapes, are mentally ill and these may contribute to the contraction of HIV. It has been reported that men who have sex with men (MSM) account for more than $50 \%$ of all new HIV/AIDS diagnoses in the United States each year, and young MSM (ages 13-24) have the highest increases in new infections. ${ }^{[12]}$ A longitudinal study with 114 young MSM (ages 16-20 at baseline) reported that increased lev- els of sensation seeking were found to significantly elevate the associations between frequency of unprotected sex and frequency of both alcohol use and drug use with partners. Follow-up result analysis reported that average rates of alcohol use moderated the association between alcohol use prior to sex and sexual risk, such that decreases in average alcohol use increased the positive association between these variables. The authors suggested that while drug use with partners increased sexual risk for all young MSM, the effects of alcohol use prior to sex were limited in low sensationseeking young MSM as well as those who are high alcohol consumers on average. ${ }^{[12]}$

Excessive alcohol consumption is another attribute that may correlates to drug abuse. Though alcohol is very similar to that of drug abuse, however, impact of alcohol can reduce a person's sense of judgment and risky sexual act. Hence excessive alcohol abuse is several times more prevalent in HIV positive people than the general population. Heavy alcohol intake, illicit drug use, and cigarette smoking, are unhealthy practices that are engaged in by many HIV-positive individuals, often as a way to manage their disease-related symptoms. ${ }^{[7]}$ In a study that examines the prevalence and characteristics of unhealthy behaviors in relation to HIV/AIDS symptoms with mean age of 42.8 years and $38.5 \%$ as female, it was reported that the mean number of years living with HIV was 9.1 years and the specific self-reported unhealthy substance-use behaviors were the use of marijuana, cigarettes, a large amount of alcohol, and illicit drugs. The authors reported that some of individuals who identified high levels of specific symptoms also reported significantly higher substance-use behaviors, including amphetamine and injection drug use, heavy alcohol use, cigarette smoking, and marijuana use. ${ }^{[7]}$

Reliable estimates of the variables are needed to determine the outcomes predictors and efficiently target limited resources for the greatest public health benefit of inpatients AIDS with mental and behavioral diseases. Our goal was to conduct a national estimate of the prevalence and factors affecting the health care outcomes of inpatient HIV/AIDS with mental and behavioral disorders by determining the variables that were more predictive of the outcomes than the traditional HIV prognostic variables.

\subsection{Theoretical framework}

To best capture the important factors that could significantly contribute to better HIV/AIDS patient care and prevention as well as future research, we utilized the Health-Related Quality of Life (HRQOL) theory. ${ }^{[13]}$ Determinants of the HRQOL were consistent with the factors that were being examined in this secondary data analysis study. The theory posits that within the level of biological factors, symptom status, functional status, general health perceptions, and overall quality of life of an HIV/AIDS patient, individual and environmental characteristics potentially have a pro- 
found impact on all the other components such as patient LOS in the theory. The HRQOL theory was used in this secondary data analyses study as a general population health status profile that provided better insight and understanding of HIV/AIDS patients at the national level, from which findings generated from the analysis could help inform practice, allocation of health care resources and health policy decisions (see Figure 1).

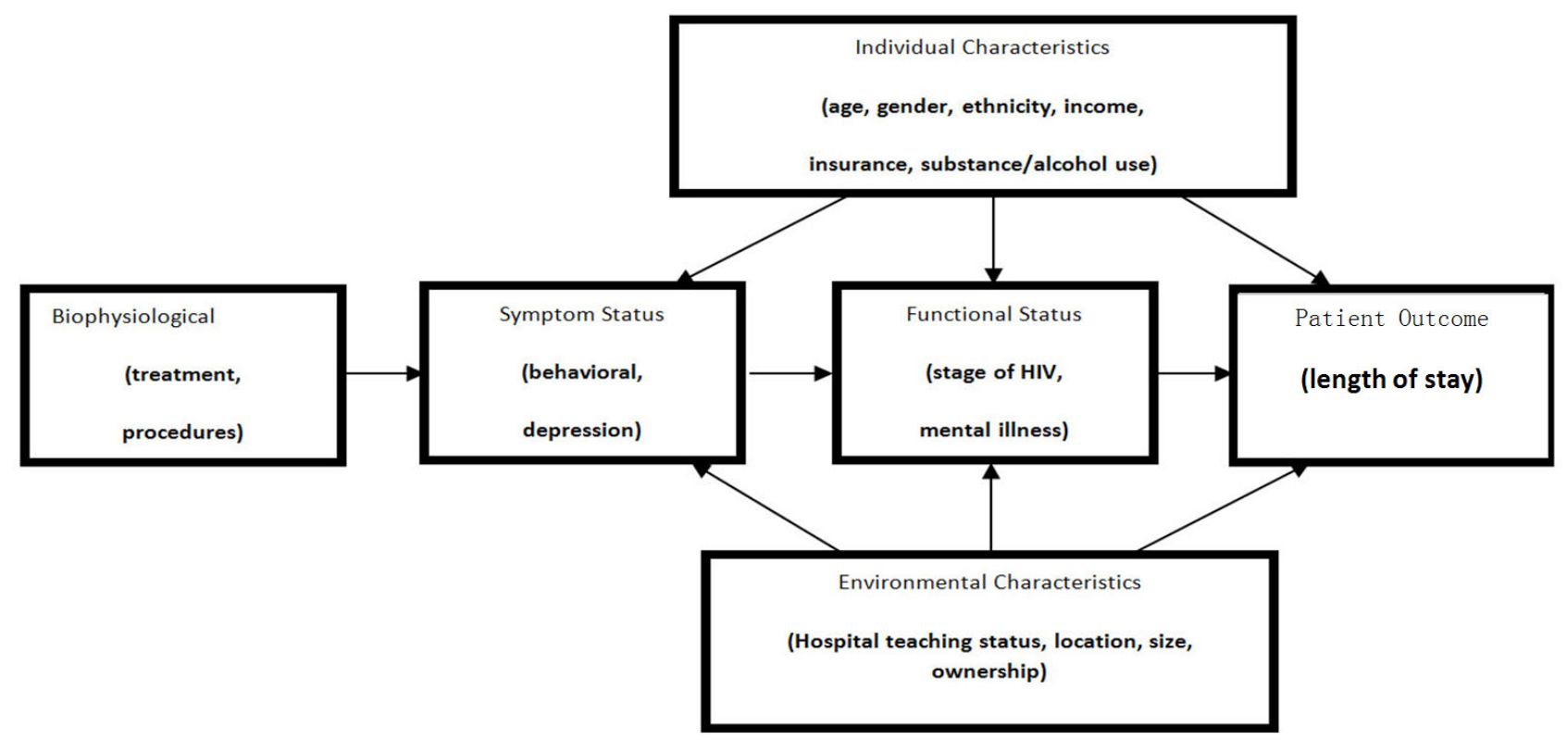

Figure 1: HRQOL Theory Concepts and Relationship with Factors in the Proposed Study Bold print suggests proposed factors in the study

\subsection{Specific aims and hypothesis}

Specific aim: Test the hypothesis that there are significant differences between patient and hospital characteristics that affect the healthcare outcomes of hospitalized HIV/AIDS in non-federal hospitals.

Research hypothesis: There will be a significant difference between patient characteristics (age, gender, ethnicity, socioeconomic status, patient location, insurance, comorbidities, treatment procedures) and hospital characteristics (location, bed size, ownership, teaching status, and hospital region) and health outcome (LOS) for hospitalized HIV/AIDS adults with mental (schizophrenia, bipolar, depression) and behavioral (alcohol and drug abuse) disorders in non-federal hospitals.

\section{Methods}

Estimate of the LOS of HIV/AIDS inpatients will be conducted by extracting and analyzing data from the Nationwide Inpatient Sample (NIS) Healthcare Costs and Utilization Program (HCUP) for 2007 and 2010. We used descriptive, bivariate, and regression analyses to test the study hypotheses. The outcome of our research could lead to a change in strategies and policies that influence the HIV prevention and treatment of adults living with HIV/AIDS and have mental illness and/or behavioral disorders in the United States.

Published by Sciedu Press

\section{Results}

We did a report on the comparison in the outcomes of the HIV/AIDS inpatients discharged in 2007 and 2010 from non- federal hospital. There was a significant difference between length of stay and age groups 30 - 39 years and 50 - 59 years for both 2007 and 2010. While the percentage of inpatients that stayed longer in the hospital were greater in 2007 for age group 30 - 39 years $(23.9 \%)$ than in $2010(18.8 \%)$, the percentage of patient stay was higher in $2010(28.7 \%)$ for the age group 50 - 59 years than in 2007 (23.8\%). Medicaid patients did also stay longer (42.2\%) in 2010 than in 2007 (37.4\%). However, the age and the ethnicity of the inpatients did not vary significantly in both 2007 and 2010 non- federal hospital admissions (see Figure 2).

The next report was on the comparison between inpatients comorbidities of the HIV/AIDS inpatients discharge in 2007 and 2010 from non- federal hospital. More HIV/AIDS inpatients' with bipolar (19.8\%) and depression (12.5\%) were admitted in 2010 than in 2007. In contrast, the inpatients with HIV/AIDs and one or more comorbidities $(21.9 \%$ and $24.1 \%$ ) respectively had more admission percentage than their counterparts in 2007 than in 2010 (see Table 1). There was no major difference between 2007 and 2010 for the medical procedures used for the inpatients admitted to nonfederal hospitals (see Table 2). 


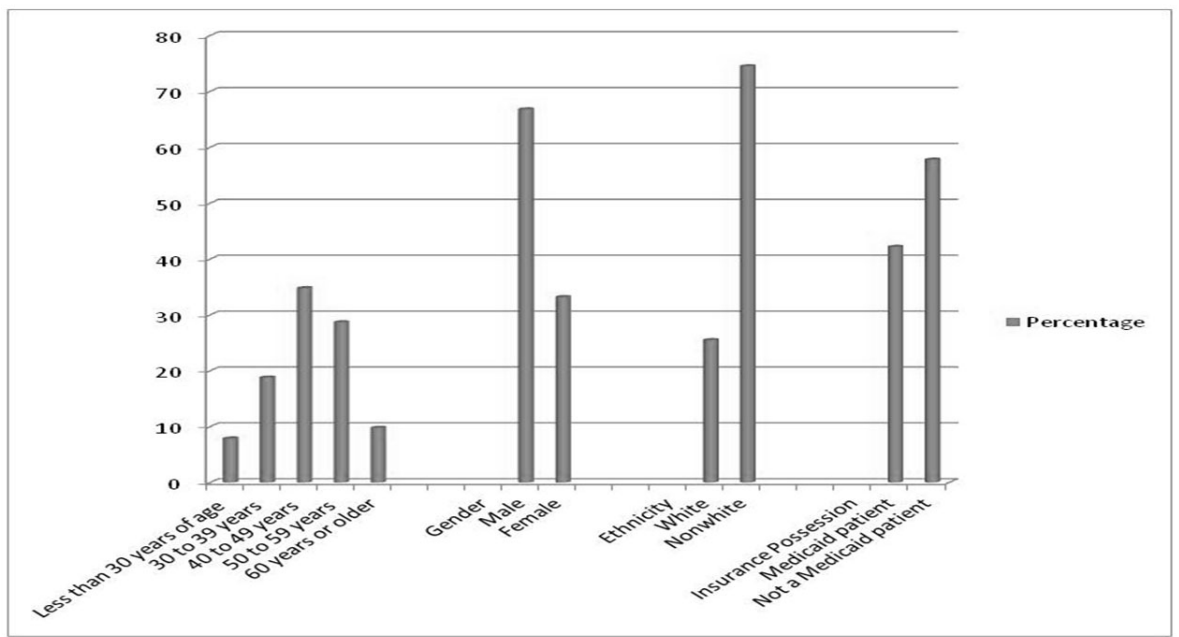

Figure 2: Percentage Distribution of Basic Patients' Characteristics in $2010(\mathrm{~N}=32,937)$

Table 1: Percentage Comparison between Inpatients Comorbidities for 2007 and 2010

\begin{tabular}{lll}
\hline Comorbidities of HIV Hospital Patients, 2007 and $\mathbf{2 0 1 0}$ & & $\mathbf{2 0 1 0}$ \\
\hline Comorbidities & $\mathbf{2 0 0 7}$ & 9.4 \\
\hline Alcohol abuse & 7.9 & 21.8 \\
Drugs abuse & 21.2 & 2.9 \\
Hepatitis b & 3.2 & 9.2 \\
Hepatitis c & 10.3 & 13.7 \\
Opportunistic illness & 14.2 & 0.2 \\
Candidiasis of the mouth & 0.2 & 12.5 \\
Depression & 9.3 & 9.8 \\
Psychosis & 6.9 & 19.8 \\
Bipolar & 14.9 & 5.1 \\
Schizophrenia & 4.1 & \\
Number of comorbidities & & 9.3 \\
None & 12.0 & 15.9 \\
1 & 21.9 & 19.6 \\
2 & 24.1 & 19.0 \\
3 & 19.6 & 14.9 \\
4 & 12.3 & 10.5 \\
5 & 6.1 & 10.7 \\
6 or more & 3.9 & 32944 \\
Number of cases & 32035 &
\end{tabular}

Table 2: Percentage Comparison between Medical Procedures Used in Treatment for 2007 and 2010

\begin{tabular}{lll}
\hline Medical Procedures Used in Treatment & $\mathbf{2 0 0 7}$ & $\mathbf{2 0 1 0}$ \\
\hline Diagnostic cardiac cath/coronary arteriography & 1.1 & 1.2 \\
Other vascular cath, not heart & 13.0 & 12.8 \\
Respiratory intubation and mechanical ventilation & 6.3 & 7.1 \\
Blood transfusion & 14.6 & 13.6 \\
Other therapeutic procedures & 9.9 & 6.8 \\
Total number of procedures (npr) & & 35.8 \\
None & 35.0 & 25.4 \\
1 & 25.5 & 14.5 \\
2 & 14.7 & 8.3 \\
3 & 8.9 & 8.5 \\
4 to 5 & 8.5 & 7.7 \\
6 or more & 7.3 & 32944 \\
Number of cases & 32035 & \\
\hline
\end{tabular}


For the hospital characteristics, the results showed that patients with HIV/AIDS were admitted more in non-teaching hospital located in the South in $2007(48.5 \%, 42.4 \%)$ than in $2010(32.0 \%, 26.9 \%)$ respectively. On the contrary, these patients were found more in large, teaching hospitals in the year $2010(70.8 \%, 73.1 \%)$ than in $2007(65.0 \%, 68.0 \%)$ respectively. Registered Nurses (RN) FTEs per 1000 patients each day increases from 2007 to 2010 while Licensed Practical Nurses' (LPNs) rate decreases from 2007 to 2010. This result showed that large teaching hospitals inpatients HIV/AIDS admission increases over the three years, supporting the increment of RN FTEs since studies have shown that positive healthcare outcomes correlates with full time employment of RNs than auxiliary LPN nurses in acute teaching hospitals. The campaign to employ BSN prepared nurses with more evidenced based research skills may have enabled this skewed result. On the other hand, inpatients' admissions decreases in the non-teaching hospitals located in the South with LPN FTEs higher in 2007 than in 2010. This above statements in which non-teaching hospitals increases in 2007, auxiliary LPNs nurses' increase in such hospitals may suggest the likelihood of professional baccalaureate prepared nurses preference of working in large teaching hospitals than non-teaching hospitals (see Table 3).

Table 3: Percentage Characteristics of Hospitals with HIV Hospital Patients, 2007 and 2010

\begin{tabular}{|c|c|c|}
\hline Hospital Characteristics & 2007 & 2010 \\
\hline \multicolumn{3}{|l|}{ Region } \\
\hline South & 48.5 & 42.4 \\
\hline North South & 51.5 & 57.6 \\
\hline Total & 100.0 & 100.0 \\
\hline \multicolumn{3}{|l|}{ Bed size of hospital } \\
\hline Small & 11.5 & 7.5 \\
\hline Medium & 23.5 & 21.7 \\
\hline Large & 65.0 & 70.8 \\
\hline Total & 100.0 & 100.0 \\
\hline \multicolumn{3}{|l|}{ Location of hospital } \\
\hline Rural & 3.9 & 3.2 \\
\hline Urban & 96.1 & 96.8 \\
\hline Total & 100.0 & 100.0 \\
\hline \multicolumn{3}{|l|}{ Hospital type } \\
\hline Teaching hospital & 68.0 & 73.1 \\
\hline Non-teaching hospital & 32.0 & 26.9 \\
\hline Total & 100.0 & 100.0 \\
\hline Number & 31807 & 32944 \\
\hline \multicolumn{3}{|c|}{ RN FTEs per 1000 patient days } \\
\hline Less than 3 per 1000 & 15.9 & 23.0 \\
\hline 3 to 3.9 per 1000 & 34.9 & 19.2 \\
\hline 4 to 4.9 per 1000 & 25.5 & 28.2 \\
\hline 5 per 1000 or more & 23.7 & 29.3 \\
\hline Total & 100.00 & 100.0 \\
\hline Number & 28976 & 29300 \\
\hline \multicolumn{3}{|c|}{ LPN FTEs per 1000 patient days } \\
\hline None & 14.6 & 16.2 \\
\hline 0.1 to 0.2 per 1000 & 37.7 & 43.7 \\
\hline 0.3 to 0.4 per 1000 & 23.2 & 27.4 \\
\hline 0.5 per 1000 or more & 24.5 & 12.8 \\
\hline Total & 100.0 & 100.0 \\
\hline Number & 29056 & 29353 \\
\hline \multicolumn{3}{|c|}{ Number aides per 1000 patient days } \\
\hline Less than 1 per 1000 & 52.4 & 56.1 \\
\hline 1 to 1.4 per 1000 & 32.8 & 29.4 \\
\hline 1.5 or more & 14.8 & 14.5 \\
\hline Total & 100.0 & 100.0 \\
\hline Number & 28991 & 28991 \\
\hline
\end{tabular}


The regression ranking results for the length of hospital stay showed that the number of medical procedures performed on a patient is the most critical factor in this model that affect the LOS of HIV/AIDS persons admitted to non-federal hospitals. Patients comorbidities, specifically mental disorders (psychosis, depression, schizophrenia, bipolar) and vascular catheterization also ranked high among the factors that affect the LOS of inpatients HIV/AIDS (see Table 4).
The result of this study has shown that patient demographics such as age, gender, ethnicity, insurance and location, size or region of the hospitals were not the most critical variables associated with longer stays in non-federal hospitals. These findings highlight the need for acute care hospitals to be fully staffed with RNs, particularly RNs with Baccalaureate (BSN) degrees for positive outcomes due to the relation of this variable with LOS.

Table 4: Ranking of Effects of Independent Variables on LOS, 2007 and 2010

\begin{tabular}{|c|c|c|c|}
\hline 2007 & & 2010 & \\
\hline Total number of procedures (npr) & 0.397 & Total number of procedures (npr) & 0.418 \\
\hline Other vascular cath, not heart & 0.154 & Total number of comorbidities & 0.164 \\
\hline Total number of comorbidities & 0.113 & Other vascular cath, not heart & 0.13 \\
\hline Bipolar & 0.104 & Psychosis & 0.095 \\
\hline Depression & 0.096 & Depression & 0.087 \\
\hline Psychosis & 0.085 & Diag. cardiac cath./coronary arteriography & 0.079 \\
\hline Schizophrenia & 0.084 & Other the rapeutic procedures & 0.079 \\
\hline Other therapeutic procedures & 0.081 & Schizophrenia & 0.078 \\
\hline Diag. cardiac cath./coronary arteriography & 0.072 & Bipolar & 0.061 \\
\hline Drugs abuse & 0.056 & RN FTEs per 1000 patient days & 0.047 \\
\hline Respiratory intubation/mechanical ventilation & 0.051 & Opportunistic illness & 0.042 \\
\hline Opportunistic illness & 0.046 & Nurse aides per 1000 patient days & 0.042 \\
\hline Blood transfusion & 0.043 & Medicaid patient & 0.038 \\
\hline White & 0.027 & Hepatitis c & 0.036 \\
\hline Urban Location of hospital & 0.023 & Respiratory intubation/mechanical ventilation & 0.035 \\
\hline Medicaid patient & 0.021 & Drugs abuse & 0.033 \\
\hline Teaching hospital & 0.021 & Southern region & 0.026 \\
\hline Hepatitis c & 0.02 & Male & 0.025 \\
\hline Bed size of hospital & 0.017 & Blood transfusion & 0.022 \\
\hline RN FTEs per 1000 patient days & 0.017 & Bed size of hospital & 0.02 \\
\hline Male & 0.016 & White & 0.018 \\
\hline Alcohol abuse & 0.012 & Urban Location of hospital & 0.018 \\
\hline Candidiasis of the mouth & 0.011 & Alcohol abuse & 0.014 \\
\hline Nurse aides per 1000 patient days & 0.011 & Hepatitis b & 0.01 \\
\hline Hepatitis b & 0.007 & Candidiasis of the mouth & 0.008 \\
\hline Southern region & 0.006 & Teaching hospital & 0.005 \\
\hline LPN FTEs per 1000 patient days & 0.004 & Age & 0.003 \\
\hline Age & 0.001 & LPN FTEs per 1000 patient days & 0.001 \\
\hline
\end{tabular}

\section{Conclusion}

This study has revealed the effects of patient and hospital characteristics as it relates to the inpatients hospitalized with HIV/AIDS in 2007 and 2010. Most importantly is the difference observed in the ranking of LOS for the HIV/AIDS inpatients. The Institute of Medicine, in its 2010 objectives called upon nursing profession to train more nurses especially for baccalaureate and other advanced degrees due to the relation of this variable with LOS, as confirmed in this report. Future research will focus on a multiple year trend of data analysis for a robust difference projection.

\section{Acknowledgements}

- This pilot study is supported by the parent grant, from Howard University “Minority Institutions' Drug Abuse Research Development Program", MIDARP- 
5R24DA02 1470-04, funded by NIH, National Institute for Drug Abuse.

- Dr. Kathy Sanders-Phillips, Principal Investigator of the Parent Grant, Howard University, College of Medicine.

- Dr. Sadaatmand Forough, Current MIDARD Direc- tor, HURD, Howard University, College of Medicine.

\section{Conflicts of Interest Disclosure}

The authors declare that there is no conflict of interest statement.

\section{References}

[1] CDC Fact Sheet. 2014. Available from: http://www. cdc.gov/hi $\mathrm{v} /$ dhap/about.html

[2] Office of the Minority Health, US Department of Health and Human Services. 2011. Available from: http://www. thebody.com/ content/art41089.html

[3] HIV symptoms. 2011. Available from: http://hivsymptomsonl ine.com/hiv-and-mental-health.html

[4] Nemoto T, Iwamoto M, Kamitani E, Morris A, Sakata M. Targeted expansion project for outreach and treatment for substance abuse and HIV risk behaviors in Asian and Pacific Islander communities. AIDS Educ Prev. 2010; 23 (2): 175-91. PMid:21517665 http://dx.doi.org/10.1521/aeap.2011.23.2.175

[5] Walkup J, Akincigil A, Hoover DR, Siegel MJ, Amin S, Crystal S. Use of Medicaid Data to Explore Community Characteristics Associated with HIV Prevalence Among Beneficiaries with Schizophrenia. Public Health Rep. 2011; 126 Suppl 3: 89-101.

[6] Vergara-Rodriguez P, Vibhakar S, Watts J. Metabolic syndrome and associated cardiovascular risk factors in the treatment of persons with human immunodeficiency virus and severe mental illness. Pharmacol Ther. 2009. 124(3): 269-78. PMid:19647020 http: //dx.doi.org/10.1016/j.pharmthera.2009.07.004

[7] Brion JM, Rose CD, Nicholas PK, Sloane R, Corless IB, Lindgren TG, et al. Unhealthy substance-use behaviors as symptomrelated self-care in persons with HIV/AIDS. Nurs Health Sci. 2011;
13(1): 16-26. http://dx.doi.org/10.1111/j.1442-2018.20 $10.00572 . \mathrm{x}$

[8] Loue S, Sajatovic M, Mendez N. Substance use and HIV risk in a sample of severely mentally Ill Puerto Rican women. J Immigr Minor Health. 2011; 13(4): 681-9. http://dx.doi .org/10.1007/s 10903-011-9452-y

[9] Meade CS, Drabkin AS, Hansen NB, Wilson PA, Kochman A, Sikkema KJ. Reductions in alcohol and cocaine use following a group coping intervention for HIV-positive adults with childhood sexual abuse histories. Addiction. 2010; 105(11): 1942-51. http: //dx.doi.org/10.1111/j.1360-0443.2010.03075.x

[10] Orwat J, Saitz R, Tompkins CP, Cheng DM, Dentato MP, Samet $\mathrm{JH}$. Substance abuse treatment utilization among adults living with HIV/AIDS and alcohol or drug problems. J Subst Abuse Treat. 2011; 41(3): 233-42. PMid:21700412 http://dx.doi.org/10.1016/j .jsat.2011.04.002

[11] Simpson CA, Xie L, Blum ER, Tucker JA. Agreement between prospective interactive voice response telephone reporting and structured recall reports of risk behaviors in rural substance users living with HIV/AIDS. Psychol Addict Behav. 2011; 25(1): 185-90. http://dx.doi.org/10.1037/a0022725

[12] Newcomb ME, Clerkin EM, Mustanski B. Sensation seeking moderates the effects of alcohol and drug use prior to sex on sexual risk in young men who have sex with men. AIDS Behav. 2011; 15(3): 565-75. http://dx.doi.org/10.1007/s10461-010-9832-7

[13] Health Related Quality of Life. 2011. Available from: http://ww w.cdc.gov/hrqol/concept.htm 